\title{
A 37-year-old man with primary antiphospholipid syndrome presenting with respiratory distress and worsening toe ischemia
}

\author{
(1) \\ Nayef M. Kazzaz, MD, ${ }^{1}$ Allecia M. Wilson, MD, ${ }^{2}$ Ruba Kado, MD, ${ }^{1}$ Geoffrey D. Barnes, MD, ${ }^{3}$ and \\ Jason S. Knight, MD, $\mathrm{PhD}^{1}$ \\ ${ }^{1}$ Division of Rheumatology, Department of Internal Medicine, University of Michigan Medical \\ School, Ann Arbor, Michigan, USA \\ ${ }^{2}$ Department of Pathology, University of Michigan Medical School, Ann Arbor, Michigan, USA \\ ${ }^{3}$ Frankel Cardiovascular Center, Department of Internal Medicine, University of Michigan \\ Medical School, Ann Arbor, MI, USA \\ Correspondence \\ Jason S. Knight, M.D., Ph.D. \\ 5520A MSRB1 \\ 1150 W Medical Center Drive, SPC 5680 \\ Ann Arbor, Ml 48109-5680 \\ Tel: 734-936-3257 \\ jsknight@umich.edu \\ Funding Sources: NMK was supported by Security Forces Hospital Program, Ministry of \\ Interior, Riyadh, Saudi Arabia. GDB was supported by the NIH grant T32-HL007853. JSK was \\ supported by career development awards from the NIH (K08AR066569), the Burroughs \\ Wellcome Fund, the Arthritis National Research Foundation, and the Rheumatology Research \\ Foundation. The authors have no conflicts of interest to disclose.
}

This is the author manuscript accepted for publication and has undergone full peer review but has not been through the copyediting, typesetting, pagination and proofreading process, which may lead to differences between this version and the Version record. Please cite this article as doi:10.1002/ acr.23168. 


\section{CASE PRESENTATION}

\section{History of present illness}

This is a 37-year-old man with a history of primary antiphospholipid syndrome (APS), with clinical manifestations including recurrent venous thrombosis, lower-extremity ulceration, and toe ischemia. He also had history of thrombocytopenia and seizure disorder. Labs were consistent with APS including prolonged dilute Russell's viper venom time (dRVVT), which was resistant to phospholipid quenching, as well as an abnormal hexagonal phase phospholipid neutralization assay. He also had high levels of both $\lg G$ anticardiolipin and IgG anti- $\beta_{2} \mathrm{GPI}$ (both consistently $>40 \mathrm{GPL}$ ). All of these labs were durably positive over many years.

The patient presented with a three-day history of dyspnea on exertion, now occurring at rest on the day of presentation. He denied productive cough or hemoptysis. He denied chest pain, nausea, vomiting, abdominal pain, change in bowel habits, or dysuria. He denied arthralgia, photosensitivity, Raynaud's phenomenon, ocular symptoms, mucosal ulceration, alopecia, or pleurisy. He denied rash, weakness, or paresthesia. He did endorse worsening bilateral foot pain for about one month prior to presentation, with the pain especially severe on the right. He denied typical symptoms of claudication. For 2 days prior to admission, the pain had been especially severe: present at rest, dull in nature, and non-radiating.

\section{Past medical history and medications}

The APS diagnosis was made at the age of 22, when he presented with an extensive left lower extremity deep venous thrombosis (DVT) event involving the left common femoral, popliteal, and peroneal veins. He had two recurrences in the same extremity within two years, both apparently in the setting of sub-therapeutic anticoagulation with warfarin.

Around age 30 , he developed intermittent discoloration of his feet, more notable on the right, as well as hypocomplementemia and thrombocytopenia: C3 of 74 (normal range 83-240 mg/dl),

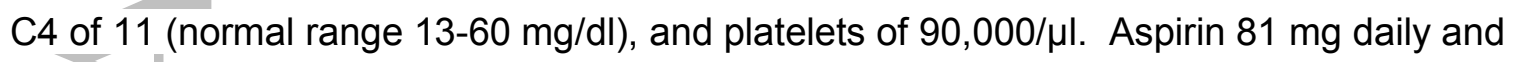
hydroxychloroquine $400 \mathrm{mg}$ daily were added to his warfarin therapy. The hydroxychloroquine was added by his rheumatologist given the hypocomplementemia, although there were no symptoms beyond these lab abnormalities to suggest a diagnosis of lupus. At this time, his hematologist also began monitoring and adjusting his warfarin dosing based on chromogenic factor $X$ assay, rather than the INR, given concern that the lupus anticoagulant was interfering with INR monitoring. The clinical and lab abnormalities all normalized with these various 
medication adjustments. Also around this time, CT angiography of the lower extremities demonstrated peripheral arterial disease, moderate-to-severe in the distal right common iliac artery and extending to the proximal right external iliac artery. Mild narrowing of the distal left common iliac artery was also present. Regarding risk factors for atherosclerosis in this 30-yearold man, there was no evidence of dyslipidemia or human immunodeficiency virus on multiple lab tests. The patient had also never been exposed to regular corticosteroids. Transthoracic echocardiography at that time showed mild mitral regurgitation with no evidence of vegetation on good quality imaging.

At age 31, he developed tonic-clonic seizures, which were subsequently well controlled with levetiracetam. Migraine headaches ensued and persisted. His doctors felt that both the seizures and migraine headaches could be attributable to APS, but there was no definitive proof.

At age 35, he developed a difficult-to-heal left lateral malleolar ulceration, first emerging after minor trauma. Given concerns about fluctunating INR testing and an inability to obtain chromogenic factor $X$ levels locally, he was switched by his local physicians to the factor Xa inhibitor rivaroxaban. It appears that rivaroxaban was chosen over low-molecular weight heparin primarily because of patient preference. The ulceration was an on-and-off issue for the next two years, and was managed primarily by the wound clinic. Although the location was a bit unusual, the prevailing opinion was that the ulcer was based in venous stasis more so than arterial insufficiency. His course was otherwise stable. There were no new thrombotic events or notable lab abnormalities (such as hypocomplementemia or thrombocytopenia). His seizure disorder was stable. He continued to work.

Although the patient lacked a symptom history to suggest a concomitant diagnosis of lupus (no arthritis, serositis, glomerulonephritis, or lupus-specific rash), "lupus labs" were obtained from time to time over the years. These revealed borderline positive testing for antinuclear antibody by HEp-2 assay and anti-double-stranded DNA by radioimmunoassay (anti-dsDNA, see below for more details). Extractable nuclear antigen testing was consistently negative for anti-SSA, anti-SSB, anti-RNP, anti-Sm, and-SmRNP, anti-chromatin, anti-RiboP, anti-centromere, antiScl70, and anti-Jo-1. Complement levels tended to be normal with the exception mentioned above. 


\section{Social and family history}

There was no family history of systemic autoimmunity, inflammatory arthritis, coagulation disorders, cardiovascular disease, or malignancy. He was a university graduate, employed, and married with two children. He denied smoking or the use of illicit drugs, and reported drinking alcohol occasionally.

\section{Physical examination}

Exam on presentation to the hospital was notable for tachycardia (129 beats per minute), tachypnea (36 breaths per minute), hypoxemia ( $83 \%$ on room air), and fever $\left(38.5^{\circ} \mathrm{C}\right)$.

Pulmonary exam demonstrated decreased breath sounds and coarse crackles at the bilateral bases. On cardiovascular exam, normal first and second heart sounds were noted with no appreciable rubs, gallops, or murmurs. Dorsalis pedis, posterior tibialis, and popliteal artery pulses were absent bilaterally. Femoral pulses were faint. He was noted to have a $2 \mathrm{~cm} \times 3 \mathrm{~cm}$ left lateral malleolar ulceration that was stable compared to exam one month prior. Additionally, the right first, second, and third toes demonstrated evidence of necrosis, compared to pinkish discoloration on recent outpatient exams.

\section{Lab testing and radiology}

His historical labs were notable for a prolonged dRVVT at 146.6 seconds (normal <42.9), dRVVT ratio 2.1 (essentially an assessment of phospholipid quenching, normal <1.19), and hexagonal phospholipid neutralization assay prolonged at 144 seconds (normal <7.9). While he was on warfarin at the time of this testing, the hexagonal assay involves mixing 1:1 with normal plasma, and prolongation due to factor deficiency is therefore unlikely. IgG anticardiolipin was $>100$ (normal <22 GPL), and IgG anti- $\beta_{2}$ GPI was >100 (normal <20 SGU). Additionally, antinuclear antibody (ANA) testing was positive at a titer of 1:80 by HEp-2 immunofluorescence assay, with a speckled pattern. Over the years, transient hypocomplementemia was once present (as above). He had also had slight elevations in anti-dsDNA antibodies in the 30s (normal range $<7.0 \mathrm{IU} / \mathrm{mL}$ ). Urinalysis was typically bland through the years, albeit with a mildly elevated urine protein:creatinine ratio of 0.26 (normal range $<0.18$ ).

In the acute setting, laboratory results were notable for total white blood cell count of $15,000 / \mu l$ with neutrophilia of $12,900 / \mu \mathrm{l}$, new normochromic normocytic anemia (hemoglobin $9.5 \mathrm{~g} / \mathrm{dl}$, decreased from a baseline of $13 \mathrm{~g} / \mathrm{dl}$ ), and new thrombocytopenia (platelets 68,000/ $\mathrm{ll}$, decreased from a recently normal baseline). Peripheral blood smear did not show evidence of 
microangiopathy. Haptoglobin, ferritin, fibrinogen, and D-dimer were all normal. A comprehensive metabolic panel was notable for newly elevated creatinine of $1.64 \mathrm{mg} / \mathrm{dl}$, blood urea nitrogen of $40 \mathrm{mg} / \mathrm{dl}$, and a slight increase in aspartate aminotransferase from 32 to 57 IU/I. Lactate was normal on presentation. Urinalysis was bland with no dysmorphic red blood cells. Urine protein-creatinine ratio showed a slight increase from 0.26 to 0.46 (normal <0.18). The erythrocyte sedimentation rate (ESR) was $53 \mathrm{~mm} /$ hour, and the C-reactive protein (CRP) was $15.2 \mathrm{mg} / \mathrm{dl}$ (normal <0.6). Both of these tests had been recently normal. Complements C3 and C4 were low at $59 \mathrm{mg} / \mathrm{dl}$ and $5 \mathrm{mg} / \mathrm{dl}$, respectively. Anti-dsDNA was positive at a level of $65 \mathrm{lU} / \mathrm{ml}$ (the hospital laboratory had recently changed from a radioimmunoassay to an ELISAbased assay, with 30-75 on the new assay considered "borderline positive"). Troponin was elevated at $12.77 \mathrm{ng} / \mathrm{mL}$ (normal range $<0.29$ ), trending down to $9.86 \mathrm{ng} / \mathrm{mL}$ over the next 48 hours. Blood cultures (both bacterial and fungal) were negative.

Portable chest X-ray demonstrated bilateral patchy opacities. Chest CT angiography (Figure 1) showing extensive multifocal patchy and ground-glass airspace opacities most pronounced at the lung bases where there was partial consolidation, left greater than right. There was no evidence of pulmonary embolism. Electrocardiogram showed sinus tachycardia, possible left atrial enlargement, and RSR' or QR pattern in V1 suggestive of right ventricular conduction delay. Transthoracic echocardiogram showed a new (as compared to a study six years prior) and severe decrease in left and right ventricular function, a dilated inferior vena cava, and worsening mitral regurgitation (previously mild, now moderate). Right ventricular systolic pressure was estimated at $33 \mathrm{mmHg}$ with right atrial pressure of $10 \mathrm{mmHg}$; there was no evidence of tricuspid valve abnormality. Abdominal and retroperitoneal ultrasounds with Doppler were normal. Bilateral ankle-branchial indices were normal; however, an abnormality was noted on toe-brachial indices.

\section{CASE SUMMARY}

This is a 37-year-old man, who carries a diagnosis of primary APS with presumed associations including: recurrent deep vein thrombosis complicated by post-thrombotic syndrome and ulceration; lower extremity atherosclerotic disease predisposing to toe ischemia; episodic thrombocytopenia; and seizure disorder. At the time of presentation, he was being treated with rivaroxaban, aspirin, and hydroxychloroquine. He presented with dyspnea, fever, hypoxemia with bilateral pulmonary ground glass opacities, worsening toe ischemia, systolic cardiac failure, 
renal failure, normochromic normocytic anemia, and thrombocytopenia. There was no evidence of hemolysis or disseminated intravascular coagulation, as demonstrated by normal haptoglobin and lack of schistocytes on peripheral blood smear.

\section{DIFFERENTIAL DIAGNOSIS}

\section{Catastrophic antiphospholipid syndrome (CAPS)}

APS is an autoimmune disease characterized by thrombosis and pregnancy loss (1). The catastrophic variety of APS (CAPS) is diagnosed in the setting of multi-organ involvement, typically with microvascular thromboses on biopsy (2). A definitive diagnosis is often only made in hindsight as histological confirmation of microvascular thrombosis is typically lacking at the time of presentation (3). Here, there is indication of multiple compromised organs, including the hypoxemic respiratory failure, rising creatinine, rising troponin with reduction in cardiac function, and toe ischemia. Pointing away from CAPS is the absence of definitive microthromboses (although with the caveat that such specimens are often difficult to obtain in critically ill patients). Further, some patients with CAPS will present with hemolysis and even microangiopathy, which this patient does not have.

\section{APS-associated diffuse alveolar hemorrhage}

The development of acute hypoxemia in an APS patient, hemoglobin drop, and new infiltrate on imaging should raise suspicion for diffuse alveolar hemorrhage. This manifestation has been described in both primary APS and CAPS (4-6). Interestingly, the pathophysiology of diffuse alveolar hemorrhage in APS is typically of inflammatory vasculitis/capillaritis, rather than thrombosis, even in patients with primary $\operatorname{APS}(5,6)$. Hemoptysis was absent here, although that will be the case for many cases of diffuse alveolar hemorrhage. Diffuse alveolar hemorrhage is of course a rare entity and there may be other explanations for the hemoglobin drop and lung infiltrate in this patient.

\section{Lupus flare}

Despite a historical diagnosis that had been labeled as "primary APS," it is warranted to assess for overlapping systemic autoimmunity, with lupus being the most common culprit.

Thrombocytopenia and worsening hypocomplementemia both raise suspicion for lupus; however, these can also be seen at modest levels in primary APS (7). Diffuse alveolar 
hemorrhage can be seen in lupus, with secondary APS and thrombocytopenia as recognized risk factors for that complication (8). Renal disease could be based in lupus glomerulonephritis, although this is unlikely in the absence of active renal sediment and with a negligible amount of proteinuria. Further, his physicians had never diagnosed him with lupus, given the absence of clinical signs and symptoms (as discussed in detail above).

\section{Infectious or Libman-Sacks endocarditis}

The worsening of mitral regurgitation raises suspicion for endocarditis. Further, primary APS is regularly associated with valvular vegetations, similar to those seen in patients with lupus $(7,9)$. Valvular vegetations can precipitate embolic phenomena (10), including ischemic events (11). The worsening of renal function could be the result of either frank embolic disease or endocarditis/immune-complex-mediated glomerulonephritis. The foot pain and toe necrosis could be secondary to embolism. Negative blood cultures point away (although do not rule out) infectious endocarditis. There were also no stigmata of peripheral embolic disease such as Janeway lesions or Osler nodes. While transthoracic echocardiogram was negative, transesophageal echocardiogram (which would have been more definitive) was not performed.

\section{Thrombotic thrombocytopenic purpura (TTP)}

With new anemia, thrombocytopenia, and renal failure, TTP can be considered. However, with no evidence of microangiopathy on blood smear, or hemolysis by biochemical parameters, this becomes less likely $(4,12)$.

\section{Sepsis/community acquired pneumonia}

Infectious pulmonary infiltrates culminating in sepsis could explain the presentation. The worsening toe ischemia could potentially stem from poor perfusion in the setting of systolic heart failure. Infection is also well known to trigger CAPS (13). Anemia and thrombocytopenia could result from disseminated intravascular coagulation in the setting of sepsis, although the normal D-dimer and fibrinogen make this less likely.

\section{HOSPITAL COURSE}

The patient's hypoxemic respiratory failure worsened, requiring intubation. He developed hypotension that was initially reversible with intravenous fluid. He received 
piperacillin/tazobactam, vancomycin, and azithromycin as treatment for suspected pneumonia and sepsis. From a rheumatologic perspective, he was managed as a possible case of CAPS with pulse-dose corticosteroids (methylprednisolone $1000 \mathrm{mg} \mathrm{x2}$ ), heparin infusion, and plasma exchange (3). Cultures obtained by mini broncho-alveolar lavage culture were negative. The mini broncho-alveolar was not sufficient to prove or disprove diffuse alveolar hemorrhage (i.e., classical assessment by serial lavage); that assessment was not performed for fear of decompensating the patient. On the third day of admission, he was improving with minimal ventilator requirements (fraction of inspired oxygen 35\%, positive end-expiratory pressure 5). He then developed sudden drop in systolic blood pressure requiring norepinephrine infusion. He developed ectopic beats, and amlodipine was added. Cardiac arrest occurred within one hour of the initial blood pressure drop. He could not be resuscitated.

\section{AUTOPSY}

The heart was enlarged in size at 630 grams (normal mean 350 grams), with broad areas of fibrosis seen both grossly and microscopically (Figure 2). Although no acute ischemia was noted grossly, there were multiple areas of microscopic ischemia (Figure 2). Coronaries were significantly and chronically stenosed with complete occlusion of the proximal left anterior descending artery and $90 \%$ of the left circumflex proximally. Microscopically, there were diffuse atheromatous plaques with chronic inflammatory changes including lymphocytes infiltrating the adventitia of all three major coronary arteries (Figure 3). The infiltrates were predominantly comprised of $\mathrm{CD}^{+}$lymphocytes. Two firm orange-tan vegetations were present on the mitral valve $(0.5 \mathrm{~cm} \times 0.4 \mathrm{~cm}$ and $0.3 \mathrm{~cm} \times 0.2 \mathrm{~cm})$, along with one friable pink-tan vegetation on the aortic valve $(1.5 \mathrm{~cm} \times 1 \mathrm{~cm})$. Microscopically, the valves demonstrated sterile fibrinous vegetations with chronic inflammation (lymphocytes more so than neutrophils), which would be consistent with Libman-Sacks endocarditis (14), rather than infectious endocarditis (15). Determining the precise etiology of Libman-Sacks endocarditis (lupus versus APS) is probably not feasible, as noted in the literature (16). The distal abdominal aorta and bilateral common iliac arteries were remarkable for moderate calcific plaques.

The pulmonary parenchyma was markedly hemorrhagic and congested. This was unlikely to be explained by post-resuscitation trauma given the widespread nature of the bleeding noted macroscopically (17). Pulmonary arteries were free from thrombi. Microscopically, the lungs had extensive acute intraalveolar hemorrhage and intraalveolar macrophages (no definitive 
hemosiderin-laden macrophages were detected). Some capillaries showed surrounding acute inflammation (Figure 4). The alveolar septae had patchy fibrosis. Focal areas of intraalveolar acute inflammation were seen. Kidneys were notable for numerous sclerotic glomeruli, chronic interstitial inflammation, and tubular calcification. The renal pathology was hard to delineate further given post-mortem changes. With that caveat, there were no features to suggest active glomerulonephritis (as in lupus) or microangiopathy/intimal hyperplasia (as in APS). The interstitial inflammation was likely nonspecific and related to either antibiotics (pipercillintazobactam is notorious) or even atherosclerosis (18). Although there was no evidence of thrombi, atherosclerosis, or vasculitis in the vessels at the base of the brain, there was brownyellow mottled discoloration near the left occipital horn of the lateral ventricle indicative of old ischemia. The liver and spleen weighed 2300 grams (normal mean 1500 grams) and 320 grams (normal mean 150 grams), respectively, with no evidence of thrombi in either.

\section{DISCUSSION}

Antiphospholipid syndrome is an autoimmune disease mediated by pathogenic autoantibodies (especially to the phospholipid-binding plasma protein $\beta_{2} \mathrm{GPI}$ ), which promote thromboinflammatory outcomes by engaging cellular membranes (19). As mentioned above, the hallmarks of the disease are obstetric complications and vascular thromboses, with both arteries and veins at risk $(1,20)$. There are other associated (sometimes called "non-criteria") manifestations of APS that cannot be explained by thrombotic events (21), such as thrombocytopenia, heart valve lesions, and seizures. Further, patients with APS have an elevated risk of cardiovascular/coronary disease $(22,23)$, and accelerated atherosclerosis (24). APS has also occasionally been linked to a traditional small-vessel vasculitis, especially in the lung capillaries (5). Other "named" vasculitides that have rarely been connected to antiphospholipid antibodies in the literature include polyarteritis nodosa and Takayasu's arteritis (25-29).

Speaking more broadly, the intersection of inflammation and coagulation pathways in APS is well-recognized, with the two going hand-in-hand to promote thrombotic events. Several cell types may be activated by antiphospholipid antibodies including endothelial cells, platelets, monocytes, and neutrophils (19, 30,31). Mechanistically, antiphospholipid antibodies promote activation of both the endothelium (for example, upregulation of vascular adhesion molecules) and the complement cascade. The result is recruitment of neutrophils and monocytes (which 
may themselves be primed by antiphospholipid antibodies) to the vascular lumen, where they activate coagulation pathways and trigger thrombosis (32).

In this patient, there is coronary disease that is well beyond what would be expected based on his age and known traditional risk factors. The adventitial layer of the coronary arteries is strikingly infiltrated by lymphocytes. Traditionally, the histopathological description of atherosclerosis is for lipid deposition, followed by stages of blood vessel wall thickening and finally fibrosis (33). While inflammatory cells may infiltrate the adventitia in atherosclerosis, they especially surround the lipid-rich regions of the vessel wall (the intima) $(34,35)$. Here, the histopathology and concentration of $\mathrm{CD}^{+}$lymphocytes in the adventitia is quite compelling (and reminiscent of what might be seen in conditions like giant cell arteritis). We speculate that some element of frank coronary vasculitis contributed to this patient's vessel wall derangements, resulting in significant, progressive stenosis of both the left anterior descending and left circumflex arteries. To our knowledge, coronary vasculitis has not been previously described in APS. Of course, an important comment is that even in the absence of definite clinical or histopathological features of lupus in this patient, we cannot completely rule out overlapping or emerging lupus. For example, recent work by our group and others has shown a type I interferon signature in patients with primary APS $(36,37)$, similar to that seen in lupus, which could have implications for the emergence of lupus features over time. Having said that, his cardiovascular findings and rapid development of multi-organ failure are well out of proportion to any solid clinical evidence for lupus here, and more likely relate to his well-established diagnosis of severe APS.

Regarding other key pathologic findings, the valvular disease in this patient is characterized by fibrinous inflammation and calcification, which is typical of what has been described in APS (i.e., Libman-Sacks endocarditis) (38). The diffuse alveolar hemorrhage here seems to be driven by a capillaritis, which has also been reported in $\operatorname{APS}(5,6,39)$.

In summary, the patient had severe coronary disease, with no clear risk factors beyond the primary APS - and with pathology supporting a potential role for coronary vasculitis. The APSrelated diffuse alveolar hemorrhage was the final stressor that set in motion the events that culminated in his death. 


\section{FINAL DIAGNOSIS}

Diffuse alveolar hemorrhage in a patient with limited cardiovascular reserve attributable to severe coronary disease-with pathology supporting a potential role for coronary vasculitis.

John Wiley \& Sons, Inc.

This article is protected by copyright. All rights reserved. 


\section{REFERENCES}

1. Miyakis S, Lockshin MD, Atsumi T, Branch DW, Brey RL, Cervera R, et al. International consensus statement on an update of the classification criteria for definite antiphospholipid syndrome (APS). J Thromb Haemost. 2006;4(2):295-306.

2. Cervera R, Font J, Gomez-Puerta JA, Espinosa G, Cucho M, Bucciarelli S, et al. Validation of the preliminary criteria for the classification of catastrophic antiphospholipid syndrome. Annals of the rheumatic diseases. 2005;64(8):1205-9.

3. Kazzaz NM, McCune WJ, Knight JS. Treatment of catastrophic antiphospholipid syndrome. Current opinion in rheumatology. 2016;28(3):218-27.

4. Rodriguez-Pinto I, Espinosa G, Cervera R. Catastrophic APS in the context of other thrombotic microangiopathies. Current rheumatology reports. 2015;17(1):482.

5. Deane KD, West SG. Antiphospholipid antibodies as a cause of pulmonary capillaritis and diffuse alveolar hemorrhage: a case series and literature review. Seminars in arthritis and rheumatism. 2005;35(3):154-65.

6. Cartin-Ceba R, Peikert T, Ashrani A, Keogh K, Wylam ME, Ytterberg S, et al. Primary antiphospholipid syndrome-associated diffuse alveolar hemorrhage. Arthritis care \& research. 2014;66(2):301-10.

7. Cervera R, Piette JC, Font J, Khamashta MA, Shoenfeld Y, Camps MT, et al. Antiphospholipid syndrome: clinical and immunologic manifestations and patterns of disease expression in a cohort of 1,000 patients. Arthritis and rheumatism. 2002;46(4):1019-27.

8. Kazzaz NM, Coit P, Lewis EE, McCune WJ, Sawalha AH, Knight JS. Systemic lupus erythematosus complicated by diffuse alveolar haemorrhage: risk factors, therapy and survival. Lupus science \& medicine. 2015;2(1):e000117.

9. Gleason CB, Stoddard MF, Wagner SG, Longaker RA, Pierangeli S, Harris EN. A Comparison of Cardiac Valvular Involvement in the Primary Antiphospholipid Syndrome Versus Anticardiolipin-Negative Systemic Lupus-Erythematosus. Am Heart J. 1993;125(4):1123-9.

10. Asopa S, Patel A, Khan OA, Sharma R, Ohri SK. Non-bacterial thrombotic endocarditis. European journal of cardio-thoracic surgery : official journal of the European Association for Cardio-thoracic Surgery. 2007;32(5):696-701.

11. Turiel M, Muzzupappa S, Gottardi B, Crema C, Sarzi-Puttini P, Rossi E. Evaluation of cardiac abnormalities and embolic sources in primary antiphospholipid syndrome by transesophageal echocardiography. Lupus. 2000;9(6):406-12. 
12. Montecucco C, Di Lauro M, Bobbio-Pallavicini E, Longhi M, Caporali R, De Gennaro F, et al. Anti-phospholipid antibodies and thrombotic thrombocytopenic purpura. Clinical and experimental rheumatology. 1987;5(4):355-8.

13. Cervera R, Rodriguez-Pinto I, Colafrancesco S, Conti F, Valesini G, Rosario C, et al. 14th International Congress on Antiphospholipid Antibodies Task Force Report on Catastrophic Antiphospholipid Syndrome. Autoimmunity reviews. 2014;13(7):699-707.

14. Bulkley $\mathrm{BH}$, Roberts WC. The heart in systemic lupus erythematosus and the changes induced in it by corticosteroid therapy. A study of 36 necropsy patients. Am J Med. $1975 ; 58(2): 243-64$.

15. Lee JL, Naguwa SM, Cheema GS, Gershwin ME. Revisiting Libman-Sacks endocarditis: a historical review and update. Clin Rev Allergy Immunol. 2009;36(2-3):126-30.

16. Eiken PW, Edwards WD, Tazelaar HD, McBane RD, Zehr KJ. Surgical pathology of nonbacterial thrombotic endocarditis in 30 patients, 1985-2000. Mayo Clin Proc. 2001;76(12):1204-12.

17. Ornato JP, Ryschon TW, Gonzalez ER, Bredthauer JL. Rapid Change in Pulmonary Vascular Hemodynamics with Pulmonary-Edema during Cardiopulmonary Resuscitation. American Journal of Emergency Medicine. 1985;3(2):137-42.

18. Chade AR, Rodriguez-Porcel M, Grande JP, Krier JD, Lerman A, Romero JC, et al. Distinct renal injury in early atherosclerosis and renovascular disease. Circulation. 2002;106(9):1165-71.

19. Giannakopoulos B, Krilis SA. The pathogenesis of the antiphospholipid syndrome. The New England journal of medicine. 2013;368(11):1033-44.

20. Davies JOJ, Hunt BJ. Myocardial infarction in young patients without coronary atherosclerosis: assume primary antiphospholipid syndrome until proved otherwise. Int $\mathrm{J}$ Clin Pract. 2007;61(3):379-84.

21. Abreu MM, Danowski A, Wahl DG, Amigo MC, Tektonidou M, Pacheco MS, et al. The relevance of "non-criteria" clinical manifestations of antiphospholipid syndrome: 14th International Congress on Antiphospholipid Antibodies Technical Task Force Report on Antiphospholipid Syndrome Clinical Features. Autoimmunity reviews. 2015;14(5):401-14.

22. Soltesz P, Szekanecz Z, Kiss E, Shoenfeld Y. Cardiac manifestations in antiphospholipid syndrome. Autoimmunity reviews. 2007;6(6):379-86.

23. Cervera R. Coronary and valvular syndromes and antiphospholipid antibodies. Thrombosis research. 2004;114(5-6):501-7. 
24. Djokovic A, Stojanovich L, Stanisavljevic N, Bisenic V, Radovanovic S, Soldatovic I, et al. Does the presence of secondary antiphospholipid syndrome in patients with systemic lupus erythematodes accelerate carotid arteries intima-media thickness changes? Rheumatol Int. 2014;34(3):321-7.

25. Han BK, Inaganti K, Fahmi S, Reimold A. Polyarteritis nodosa complicated by catastrophic antiphospholipid syndrome. Journal of clinical rheumatology : practical reports on rheumatic \& musculoskeletal diseases. 2004;10(4):210-3.

26. Schoonjans R, Van Vlem B, Weyers S, De Keyser F, Praet M, Versieck J, et al. Polyarteritis nodosa and the antiphospholipid syndrome. Clinical rheumatology. 1996;15(4):410-

3.

27. Norden DK, Ostrov BE, Shafritz AB, Von Feldt JM. Vasculitis associated with antiphospholipid syndrome. Seminars in arthritis and rheumatism. 1995;24(4):273-81.

28. Yokoi K, Hosoi E, Akaike M, Shigekiyo T, Saito S. Takayasu's arteritis associated with antiphospholipid antibodies. Report of two cases. Angiology. 1996;47(3):315-9.

29. Dongola NA, Foord KD. Angiographic features associated with antiphospholipid syndrome. The British journal of radiology. 2000;73(875):1215-8.

30. Yalavarthi S, Gould TJ, Rao AN, Mazza LF, Morris AE, Nunez-Alvarez C, et al. Release of neutrophil extracellular traps by neutrophils stimulated with antiphospholipid antibodies: a newly identified mechanism of thrombosis in the antiphospholipid syndrome. Arthritis \& rheumatology. 2015;67(11):2990-3003.

31. Meng H, Yalavarthi S, Kanthi Y, Mazza LF, Elfline MA, Luke CE, et al. In vivo role of neutrophil extracellular traps in antiphospholipid antibody-mediated venous thrombosis. Arthritis \& rheumatology. 2016.

32. Lally L, Sammaritano LR. Vasculitis in antiphospholipid syndrome. Rheum Dis Clin North Am. 2015;41(1):109-23, ix.

33. Stary HC, Chandler AB, Dinsmore RE, Fuster V, Glagov S, Insull W, Jr., et al. A definition of advanced types of atherosclerotic lesions and a histological classification of atherosclerosis. A report from the Committee on Vascular Lesions of the Council on Arteriosclerosis, American Heart Association. Arteriosclerosis, thrombosis, and vascular biology. 1995;15(9):1512-31.

34. van der Wal AC. Coronary artery pathology. Heart. 2007;93(11):1484-9.

35. van der Wal AC, Becker AE. Atherosclerotic plaque rupture--pathologic basis of plaque stability and instability. Cardiovascular research. 1999;41(2):334-44. 
36. Grenn RC, Yalavarthi S, Gandhi AA, Kazzaz NM, Nunez-Alvarez C, Hernandez-Ramirez $D$, et al. Endothelial progenitor dysfunction associates with a type I interferon signature in primary antiphospholipid syndrome. Annals of the rheumatic diseases. 2016.

37. van den Hoogen LL, Fritsch-Stork RD, Versnel MA, Derksen RH, van Roon JA, Radstake TR. Monocyte type I interferon signature in antiphospholipid syndrome is related to proinflammatory monocyte subsets, hydroxychloroquine and statin use. Annals of the rheumatic diseases. 2016.

38. Garcia-Torres R, Amigo MC, de la Rosa A, Moron A, Reyes PA. Valvular heart disease in primary antiphospholipid syndrome (PAPS): clinical and morphological findings. Lupus. 1996;5(1):56-61.

39. Asherson RA, Greenblatt MA. Recurrent alveolar hemorrhage and pulmonary capillaritis in the "primary" antiphospholipid syndrome. Journal of clinical rheumatology : practical reports on rheumatic \& musculoskeletal diseases. 2001;7(1):30-3.






\section{FIGURE LEGENDS}

Figure 1 Multifocal patchy and ground-glass airspace opacities. These are most pronounced at the lung bases where there is partial consolidation, left greater than right.

Figure 2 Left ventricle, microscopic view. Left panel: broad areas of dense collagenous scar. Right panel: subacute changes (in response to ischemia) with granulation tissue and early collagen deposition.

Figure 3 Left anterior descending artery, microscopic view. A, Focal complete luminal occlusion with dense intramural inflammatory response, especially prominent at the mediaadventitia interface. There is a predominance of $\mathrm{CD}^{+}$lymphocytes (B), with less CD8 ${ }^{+}$ lymphocytes (C) and natural killer (NK) cell (D) infiltration.

Figure 4 Pulmonary capillaritis, microscopic view.



John Wiley \& Sons, Inc.

This article is protected by copyright. All rights reserved. 




Figure 1



$82 \times 55 \mathrm{~mm}(300 \times 300 \mathrm{DPI})$

John Wiley \& Sons, Inc.

This article is protected by copyright. All rights reserved. 




Figure 2

$162 \times 61 \mathrm{~mm}(300 \times 300$ DPI $)$

John Wiley \& Sons, Inc.

This article is protected by copyright. All rights reserved. 




Figure 3

$152 \times 114 \mathrm{~mm}(300 \times 300 \mathrm{DPI})$

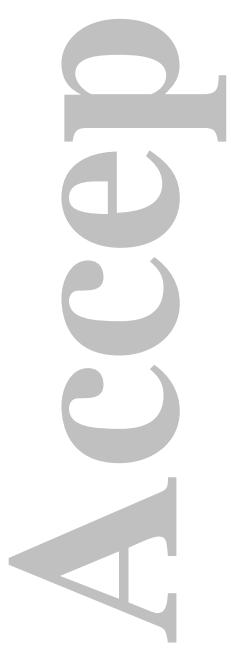

John Wiley \& Sons, Inc.

This article is protected by copyright. All rights reserved. 


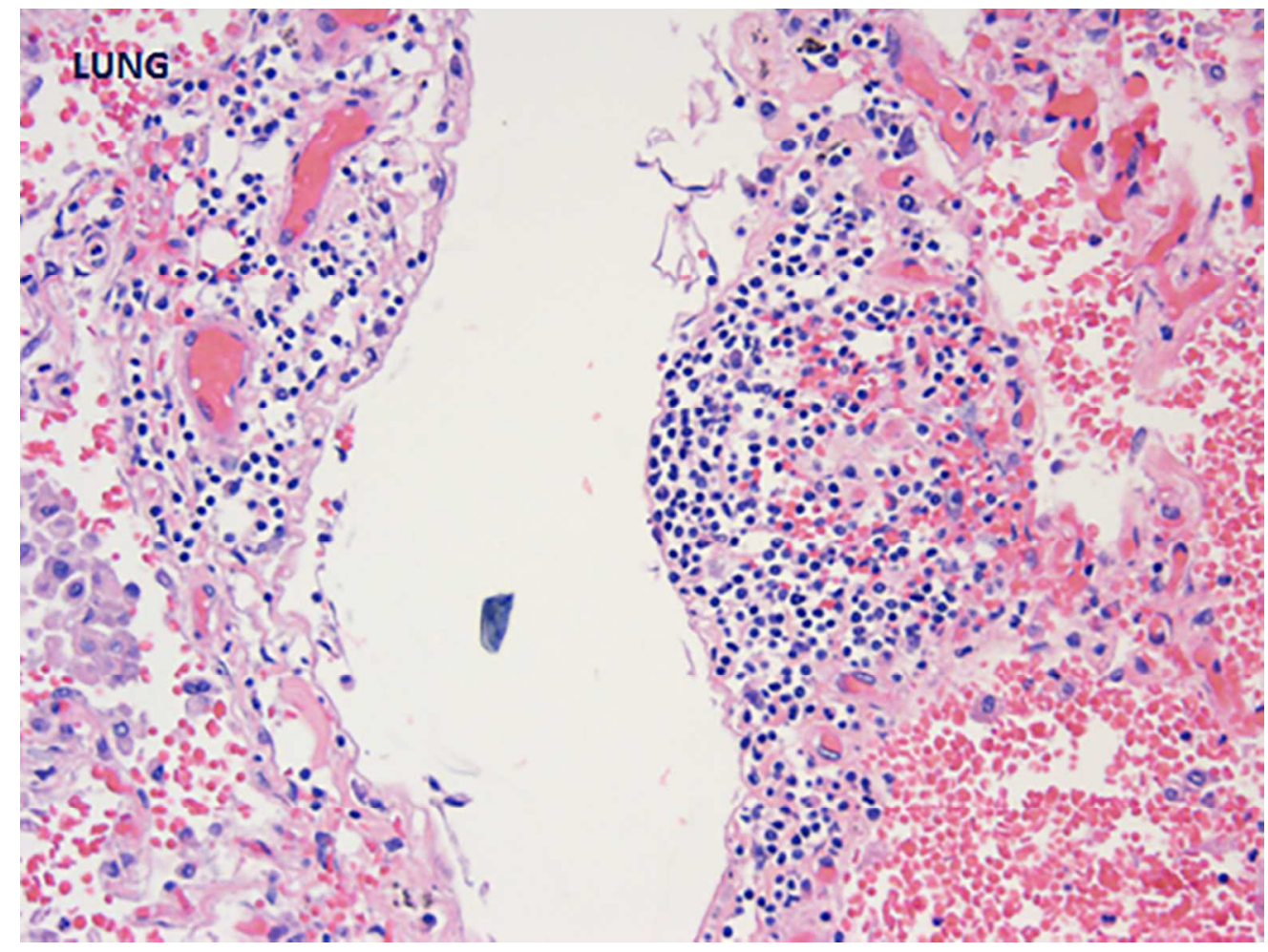

Figure 4

$82 \times 61 \mathrm{~mm}(300 \times 300$ DPI $)$



John Wiley \& Sons, Inc.

This article is protected by copyright. All rights reserved. 